\title{
Functional connectivity analysis of the neural bases of emotion regulation: A comparison of independent component method with density-based k-means clustering method
}

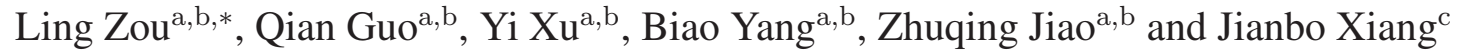 \\ ${ }^{a}$ School of Information Science and Engineering, Changzhou University, Changzhou, Jiangsu, China \\ ${ }^{\mathrm{b}}$ Changzhou Key Laboratory of Biomedical Information Technology, Changzhou, Jiangsu, China \\ ${ }^{\mathrm{c}}$ Changzhou NO.2 People's Hospital Affiliated with Nanjing Medical University, Changzhou, Jiangsu, \\ China
}

\begin{abstract}
Functional magnetic resonance imaging (fMRI) is an important tool in neuroscience for assessing connectivity and interactions between distant areas of the brain. To find and characterize the coherent patterns of brain activity as a means of identifying brain systems for the cognitive reappraisal of the emotion task, both density-based k-means clustering and independent component analysis (ICA) methods can be applied to characterize the interactions between brain regions involved in cognitive reappraisal of emotion. Our results reveal that compared with the ICA method, the density-based k-means clustering method provides a higher sensitivity of polymerization. In addition, it is more sensitive to those relatively weak functional connection regions. Thus, the study concludes that in the process of receiving emotional stimuli, the relatively obvious activation areas are mainly distributed in the frontal lobe, cingulum and near the hypothalamus. Furthermore, density-based k-means clustering method creates a more reliable method for follow-up studies of brain functional connectivity.
\end{abstract}

Keywords: Functional connectivity, cognitive reappraisal, density-based k-means clustering method, ICA

\section{Introduction}

Functional magnetic resonance imaging (fMRI) is an emerging imaging technology for the current brain research. Generally, fMRI can get a magnetic resonance image with high spatial resolution of millimeters. In addition, fMRI is more advantageous as it is non-invasive, non-radioactive and repeatable. It has been widely used for theoretical and clinical research in the fields of cognitive science, psychology and brain science [1].

According to the Gross of emotion regulation process model, cognitive reappraisal, as antecedentfocused strategies, occurred in the early phase of mood changes, mainly by changing the understanding of emotional events changed the understanding of the personal meaning of emotional events to reduce the emotional response [2]. Brain functional connectivity is one of the effective modes to describe the

\footnotetext{
${ }^{*}$ Corresponding author: Ling Zou, School of Information Science and Engineering, Changzhou University, Changzhou, Jiangsu, China. Tel.: +86 13813583689; E-mail: zouling@cczu.edu.cn.
} 
collaborative work between various brain regions. It firstly emerged in the concept of an electrophysiological study. Functional connectivity is described as the temporal correlation of the brain regions which separated on the space. The study of functional connectivity is intended to detect and characterize the coherent patterns of brain activity as a means of identifying brain systems [3]. It can also provide explanation and tips for each brain region in the task. For example, studies have found that reactions in episodic memory can be predicted by the functional connectivity of hippocampus [4].

Methods of functional connectivity analysis through fMRI can be categorized into two classes: modelbased methods and data-driven methods. Data-driven methods, which require no prior knowledge, effectively overcome the uncertainty of selecting seeds zone. For brain functional connectivity analysis, data-driven methods have two forms: decomposition-based methods and clustering-based methods. Examples of the decomposition-based methods are principal component analysis (PCA), singular value decomposition (SVD) and independent component analysis (ICA) [5-7]. These methods usually try to explicit the original fMRI data set as a linear combination of basis vectors or statistically independent components. However, the problem for them is the meaningful decomposed components are notoriously difficult to select. Clustering-based method includes Fuzzy clustering analysis (FCA) and hierarchical clustering analysis (HCA) [8,9], but the major drawback for them is that they are supervised by the selection of the initial clustering center.

ICA is developed from blind source separation technology. Depending on the separation of variables (i.e., temporal or spatial independent), ICA can be characterized as either temporal ICA (tICA) or spatial ICA (sICA). Generally, sICA is more widely used for analysis of fMRI data. It can decompose an original data into numerous components that have the maximized independence [10]. In addition, ICA can effectively decompose various statistical independence components, such as non-Gaussian functional signals, and all kinds of noises. However, the results alone are difficult to be directly interpreted, because of the spatiotemporal correlations of spontaneous blood oxygenation level-dependent (BOLD) signals. Compared with the pathological study, changes of brain function caused by emotion regulation are not very obvious that the traditional method such as ICA cannot capture all the details. In order to overcome this, density value has been introduced to k-means clustering methods. It has a fundamental difference compared with other methods: it is based on the density instead of the distance. The proposed method not only successfully suppresses the impact of the individual isolated points but also greatly improves the clustering accuracy and stability. It can also group the brain voxels into different clusters that represent brain default mode networks (DMNs).

In this work, we propose a density-based k-means clustering algorithm for analyzing fMRI data set and extracting correlation patterns for DMNs representation. The proposed algorithm can be applicable to the data set from normal subjects with cognitive reappraisal experiment. The concept of density in order to optimize the initial clustering center selection, because it is difficult to get the representative dispersion point when selecting the initial cluster centers by regular k-means method. The results are compared with those obtained using ICA to evaluate the effectiveness of the proposed method.

\section{Materials and methods}

\subsection{Subjects}

Eleven healthy, right-handed participants (six men and five women, aged from 19-24 with a mean of 22.09) are involved in this study. Participants have normal or corrected normal vision without a history of neurological, medical or psychiatric disorders. All participants provided written informed consent 


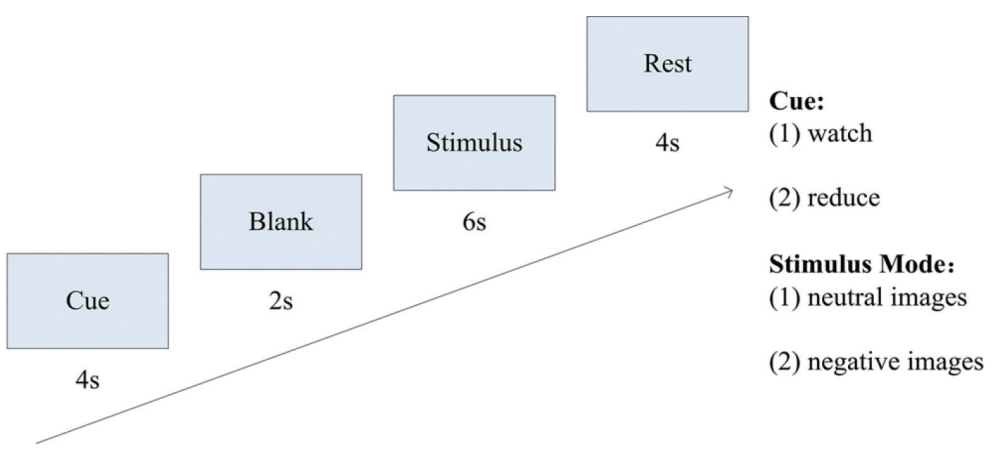

Fig. 1. The experimental paradigm of cognitive reappraisal.

to be part of the experiment, which is approved by the local ethics committee (Changzhou University, Changzhou, China).

\subsection{Task procedure}

In this study, a task involving active, voluntary regulation of negative emotion by cognitive reappraisal is employed. The experimental stimuli include 120 pictures, which are chosen from the International Affective Picture System (IAPS). Among them, 80 pictures are unpleasant scenes (valence: $M=2.85$, $\mathrm{SD}=1.65$ ). The other 40 pictures are non-affective neutral nature scenes (valence: $\mathrm{M}=5.21, \mathrm{SD}$ $=1.25$ ). The pictures introduce both evaluation and experience of negative affect and show complex scenes. More importantly, the pictures are matched for general content.

Three conditions are considered in the task: 'watch-neutral, watch-negative and suppress-negative'. Under the watch-neutral or watch-negative condition, participants are instructed to behave naturally (not try to change) the emotional state elicited by the pictures. In the suppress-negative condition, participants are instructed to voluntarily decrease the intensity of their negative impacts by using the cognitive strategy of reappraisal. They are asked to reinterpret the content of the picture so that the picture did not elicit a negative response. The procedure for each trial is shown in Fig. 1: 1) 4 s instruction ("Watch" or "Suppress"), 2) $2 \mathrm{~s}$ blank screen, 3) $6 \mathrm{~s}$ pictures and rating of the intensity and pleasantness on a 9-point scale ( $1=$ not at all to $3=$ moderately to $9=$ extremely), 4$) 4$ s static landscape image making participants relax and stop maintaining or reappraising their emotional experience.

During scanning, participants completed 120 trials over 4 individual scans. 10 watch-neutral, 10 watch-negative and 10 suppress-negative trials are involved in each scan. The trial order is counterbalanced across scans so that every trial type follows the others with equal probability. Assignment of photos to trial types and scans is also counterbalanced across participants. E-prime is used to control stimulus presentation and response collection.

\section{3. fMRI data acquisition}

Functional MRI is conducted on a 3-T scanner (Philips Medical Systems) to acquire bloodoxygenation-level-dependent (BOLD) signal using gradient echo $\mathrm{T} 2 *$-weighted echo-planar imaging (EPI) (repetition time of $2000 \mathrm{~ms}$, echo time of $35 \mathrm{~ms}$ and flip angle of $90^{\circ}$ ). 24 continuous slices parallel to the anterior commissure-posterior commissure line are acquired per volume (field of view of $230 \mathrm{~mm} \times 182 \mathrm{~mm}$, matrix of $96 \times 74$ and voxel size of $3 \times 3 \times 3$ ). Totally, 240 volumes are acquired for each of the four runs. A T1-weighted structural image $(1 \times 1 \times 1 \mathrm{~mm})$ is also acquired for each participant at the end of the experiment. 


\section{4. fMRI data processing}

The fMRI data are processed and analyzed using SPM8 (http://www.fil.ion.ucl.ac.uk/spm/). The first 4 functional volumes of each run are discarded. The rest images are corrected for slice-timing artifacts and spatially realigned to the first image of each timeline. It is then normalized as a standard template based on the Montreal Neurologic Institute (MNI) reference brain. The data are smoothed with an $8 \mathrm{~mm}$ FWHM Gaussian kernel. In the analysis, a model of a box-car convolved with a canonical hemodynamic response function for the 6-sec trial epoch when the participants reappraised or attended as a picture is on the screen. In addition, linear drift removal and temporal filtering are done using Resting-State fMRI Data Analysis Toolkit, REST (http://www.restfmri.net/forum/). In the end, we used ICA and densitybased k-means clustering method to characterize the functional connectivity of the brain areas involved in emotion regulation.

\subsubsection{Infomax ICA}

In this paper, the cognitive reappraisal fMRI data set is characterized as a 4D matrix with one temporal and three spatial dimensions. First, the three spatial dimensions should be reshaped to one dimension. Then, the cognitive reappraisal fMRI data set can be converted into a $2 \mathrm{D}$ (time $\times$ voxel) matrix $\mathrm{X}$, where $X$ is an $m \times n$ matrix. $m$ is the number of time points and $n$ is the number of voxels. On the one hand, Infomax ICA $[10,11]$ is used to evaluate the number of independent components and calculate the correlation matrix $\mathrm{C}_{n \times n}$. Each row of $\mathrm{C}_{n \times n}$ represents the correlations of a specific voxel to all voxels. Finally, the image of whole brain activation can be extracted and the index of voxel-based aggregation index (VBAI) need to be recorded.

\subsection{2. $K$-means clustering}

$\mathrm{K}$-means clustering is a clustering method that follows hard divided guidelines. It defines each object can only be segregated into one class. The main idea is to identify cluster centers by repeating iteratively [12]. Based on the principle of higher similarity in cluster and lower similarity inter-cluster, it divides the data set of objects $n$ into k clusters. The similarity of one cluster which is calculated by the average value can be regarded as the centroid of cluster. The total Euclidean distance of objects $\mathrm{n}$ and clusters of $k$ can be calculated as Eq. (1) where $X_{j}(j=1, \ldots, n)$ means data set and $G_{i}(i=1, . ., k)$ means the number of clusters.

$$
J=\sum_{i=1}^{k} J_{i}=\sum_{i=1}^{k}\left(\sum_{j, X_{j} \in G_{i}}\left\|X_{j}-G_{i}\right\|\right)=\sum_{i=1}^{k}\left(\sum_{j, X_{j} \in G_{i}} d\left(X_{j}, G_{i}\right)\right)
$$

So, the distance between the two objects $x_{i}$ and $x_{j}$ is defined as Eq. (2) where $c$ is the number of properties of samples and $k$ is the number of clusters.

$$
d\left(x_{i}, x_{j}\right)=\sqrt{\sum_{k=1}^{c}\left(x_{i k}-x_{j k}\right)^{2}}
$$

In order to assess the similarity of internal clusters, a measurable parameter $E$ has been pulled in, which is defined as the sum of squared error between all the objects in the data set and the center of clusters. $\mathrm{E}$ can be calculated according to Eq. (3).

$$
E=\sum_{i=1}^{k} \sum_{x \in C_{i}}\left|x-\overline{x_{i}}\right|^{2}
$$


The value of E satisfies the positive correlation with similarity within a cluster. $x$ is an object in a cluster and $x_{i}$ is weighted centroid for the cluster $C_{i}$ which represents the $i t h$ cluster [13].

The process of hard k-means clustering is described as follows:

(1) Determine the number of clusters and select the initial cluster center randomly for each class.

(2) With Eq. (2), calculate the Euclidean distance between any two objects in the data sets successively, then each object will be assigned to the nearest cluster based on the minimum Euclidean distance.

(3) Calculate the sample mean of each cluster to find new centers and replace the original ones.

(4) Repeat step (2) and step (3) until the absolute value of difference between two adjacent values calculated from Eq. (3) is less than the preset threshold. Afterwards, all of the objects are divided into the specified number of clusters.

\subsubsection{Density-based k-means clustering}

K-means clustering has the advantages of scalability and efficiency, but it may be uncertain when selecting the initial cluster center. This results in degrading the quality of the final clustering results [14, 15]. An improved K-means algorithm based on destiny has been proposed to overcome this problem.

The process of density k-means clustering is described as follows:

(1) Calculate the Euclidean distance between any two objects.

(2) Calculate the density values of all objects. For an arbitrary point of object $P$, the density value for $P$ is the number of all points in a field with a specified radius $R$. That is the density based on the field $R$. Then identify the highest density object as the initial center.

(3) Calculate the Euclidean distance from each object to the initial cluster center. Then locate the object which is farthest from the initial center at the next cluster center.

(4) Repeat step (3) and calculate the remaining sample data in order to ensure all cluster centers.

The initial cluster centers are substantially uniformly distributed after the density has been calculated. As the problem of isolated points and individual empty classes still exists, the preset number of clustering should be slightly greater than the target one. Calculate the Euclidean distance of the remaining class and merge the nearest class, meanwhile, remove the worthless class and isolated points until the clustering number reaches the target value. Density-based k-means clustering effectively suppress the influence of individual isolated points, and makes the selection of initial clustering centers distribute as reasonable as possible. This will greatly improve the accuracy and stability of the cluster.

\subsection{Voxel-based aggregation index (VBAI)}

Aggregation index is originally put forward in landscape ecological studies [16]. It is class-specific and independent of landscape composition. In aggregation index, it is assumed that a class who shows the highest level of aggregation (aggregation index $=1$ ) is consisted of pixels sharing the most possible edges, while a class who has the lowest level of aggregation (aggregation index $=0$ ) shares no edges (completely segregated). The calculation of aggregation index is from a 2D matrix and then extended the computation of the aggregation index values to a $3 \mathrm{D}$ matrix. In this study, one parameter called voxel-based aggregation index (VBAI) is proposed to evaluate the quality of "meaningful" brain maps since the fMRI BOLD signals which are consist of voxels are acquired from 3D volumes. VBAI is used to quantitatively comparing and evaluating the results of the density-based k-means clustering and independent component analysis (ICA). In the cluster or independent component, it will be ruled out if the value of VBAI is below the predefined limits as these patterns cannot well describe the neural network activity. 

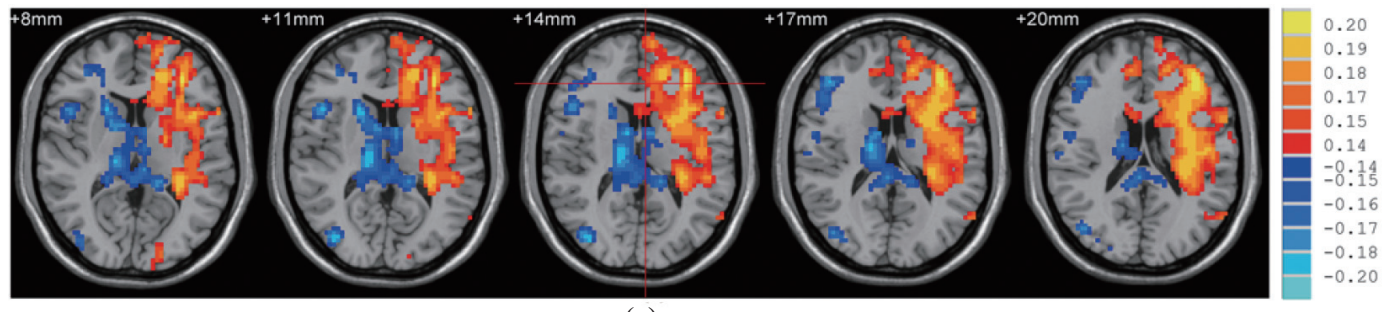

(a)

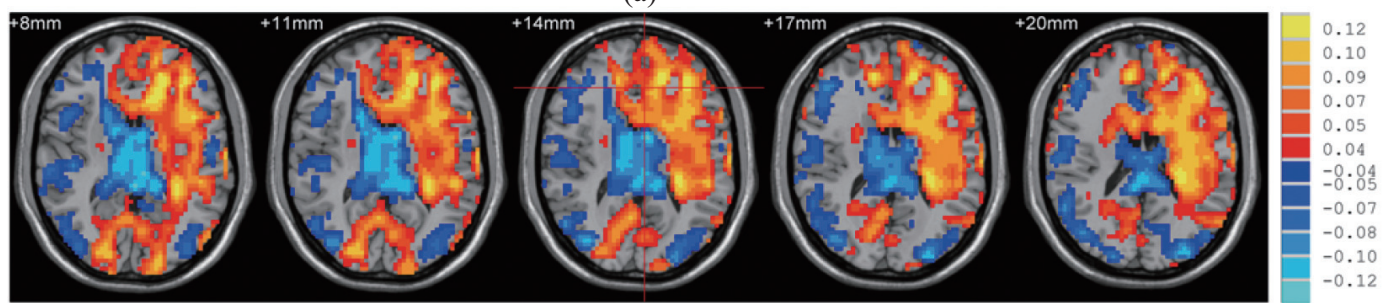

(b)

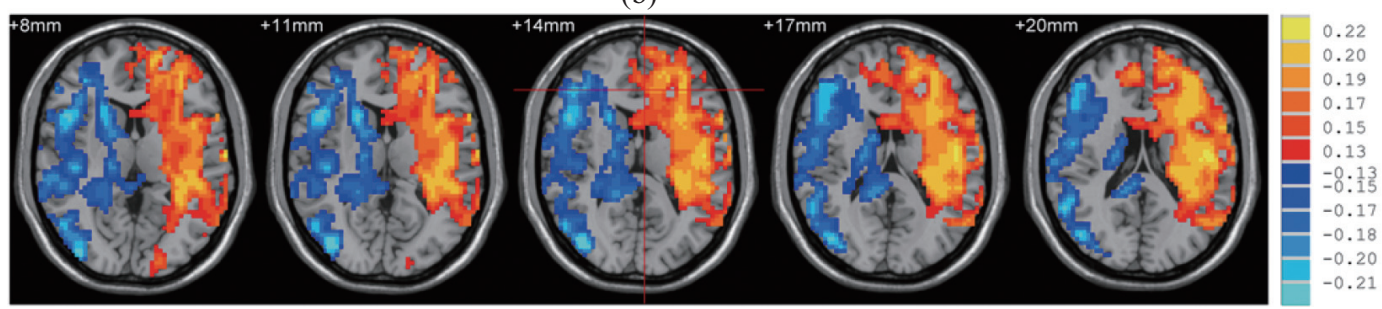

(c)

Fig. 2. The whole brain activation map obtained by density-based k-means clustering with the task of cognitive reappraisal: (a) watching the neutral images; (b) watching the negative images; (c) reducing the negative images.

\section{Results}

In recent years, many imaging experiments show that the change of mood can really affect cognition, negative emotional stimulation effect of cognitive domain mainly present in the prefrontal cortex and the anterior cingulum. These areas play an extremely important role in cognitive control [1-3].

Emotional memory is considered to be closely linked to the areas such as the cingulum cortex, limbic system and so on [17-19]. Particularly, the influence of the amygdale and hypothalamus in the limbic system is most significant. In this paper, the activation of cingulum cortex and hypothalamus has been mainly analyzed. Figures 2 and 3 show the image of whole brain activation which obtained by densitybased k-means clustering and infomax ICA in three different types of task model.

In this paper, four brain regions are mainly studied. They are Cingulate_Ant_L, Cingulate_Ant_R, Thalamus_L and Thalamus_R in the Automated Anatomical Labeling (AAL) template. From the image of whole brain activation, Fig. 2 shows the regional distribution is more concentrated than Fig. 3. Particularly, intensity of brain region activation is strongest in the task of watching negative images, the activated regions almost covering the right Posterior Cingulate Corte (PCC) and its adjacent precuneus. Correspondingly, reducing the negative images, which add the emotional self-regulation, shows more obvious and concentrated in activated brain regions; the contact between right medial prefrontal cortex (mPFC) and right PCC tend to be weaker. Contrast three activation images in Fig. 2, obviously, emotion regulation strategies can effectively suppress brain regions activation, but cannot stop absolutely. This phenomenon does conform to the definition of cognitive reappraisal. Reflected in the Fig. 2, the 
Table 1

Comparison between density-based k-means clustering and infomax ICA methods for the value of VBAI

\begin{tabular}{lcc}
\hline Stimulus type/processing mode & Density-based k-means clustering & Infomax ICA \\
\hline Watching neutral images & $0.85(0.032)$ & $0.77(0.023)$ \\
Watching negative images & $0.85(0.027)$ & $0.77(0.024)$ \\
Reducing negative images & $0.85(0.029)$ & $0.77(0.023)$ \\
\hline
\end{tabular}

${ }^{*}$ Standard deviations are presented in parentheses.
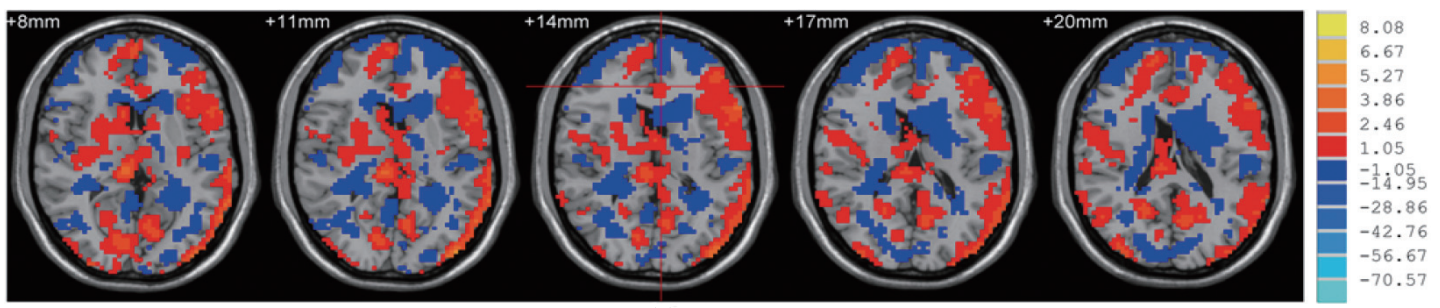

(a)
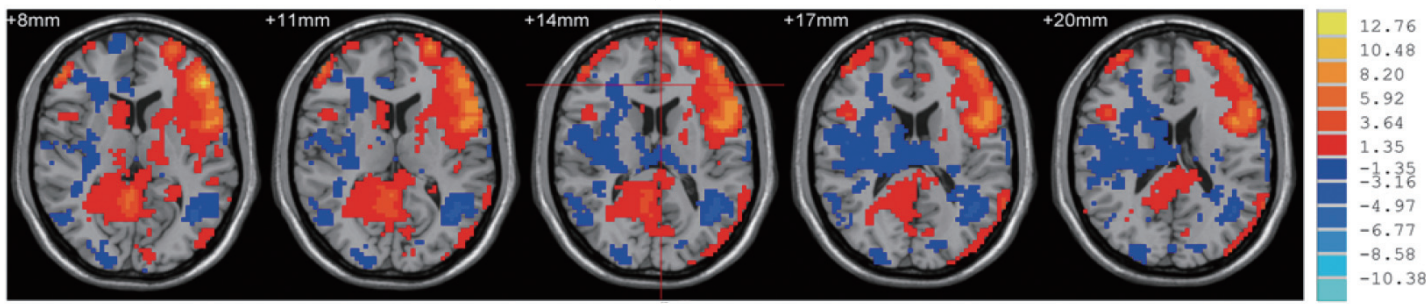

(b)
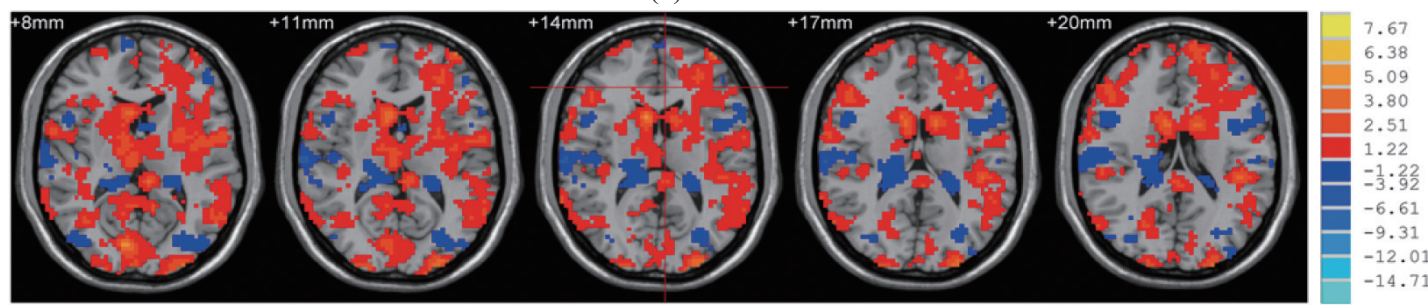

(c)

Fig. 3. The whole brain activation map obtained by infomax ICA with the task of cognitive reappraisal: (a) watching the neutral images; (b) watching the negative images; (c) reducing the negative images.

activation of watching neutral images is gentler than reducing negative images. In addition, there is also a small amount of activation in the frontal lobe and the occipital lobe, as prefrontal cortex is closely related to cognitive activities and visual information need to be processed to rely on the occipital lobe. The experimental results can be ascribed to the theory analysis.

Compared with the results from k-means method, the figures obtained by infomax ICA do not obviously activated in right $\mathrm{mPFC}$ regions. In addition, these two methods demonstrate different DMNs including bilateral anterior Cingulate Corte (ACC) and PCC, but in several regions. Especially in right ACC, k-means shows better results. The consequences of VBAI for each method are set out in Table 1 . In the comparison of the results of individual subjects and superposition of overall for both methods, the value of VBAI calculated by density-based k-means clustering is obviously higher than the other one. Therefore, compared with infomax ICA, density-based k-means clustering can find out those weaker voxel which is not easy to extract, and the activated regions are more concentrated. 


\section{Conclusion}

In this paper, we implemented ICA and density-based k-means clustering algorithms to extract emotion related brain areas with functional connectivity. According to the spatial similarity of the functional connection diagrams, we classified different functional connection diagrams into different clusters. This method can extract effectively brain areas with functional connectivity in emotion regulation process.

ICA has a very important assumption that the observed signal is made up of a plurality of uncorrelated original signals. In order to separate those independent source signals from the mixed signal, the researchers use different ICA algorithms to estimate the independent components, such as infomax ICA and Fast ICA. These models can accurately describe and clearly define the problem of the source signal, but it may not be appropriate to address the functional connectivity problems of cognitive reappraisal, which have complex relationships among different brain regions.

The density-based k-means clustering algorithm proposed in this paper is based on data-driven principle, which is not affected by prior knowledge selected by "seed zone" and can avoid effectively the bias caused by different "seed zones". This algorithm can identify a plurality of modes at one time. The results of the functional connectivity diagrams are acquired by averaging multiple correlation diagrams. Therefore, the functional connectivity diagrams show high quality. Compared with ICA method, the clustering algorithm has higher sensitivity and polymerization degree. Its characteristics of strong activation energy also ensure that certain brain areas with weak functional connectivity can also be identified correctly. The activation strength of functional connectivity diagrams actually represents the average values of the correlation coefficients among voxels, which are the strength of functional connection. From the perspective of the functional connection strength of cognitive reappraisal, it makes the results from cluster analysis method easier to be understood than other multivariate analysis methods.

\section{Acknowledgments}

This work has been partially supported by National Natural Science Foundation of China (61201096, 51307010), the Science and Technology Program of Changzhou City (CE20145055) and Qing Lan Project of Jiangsu Province.

\section{References}

[1] Mak AK, Hu ZG, Zhang JX, Xiao ZW, Lee TM. Neural correlates of regulation of positive and negative emotions: An fMRI study. Neurosci Lett. 2009; 457(2): 101.

[2] Koch K, Pauly K, Kellermann T, Seiferth NY, Reske M, Backes V, et al. Gender differences in the cognitive control of emotion: An fMRI study. Neuropsychologia. 2007; 45(12): 2744.

[3] Ertl M, Hildebrandt M, Ourina K, Leicht G, Mulert C. Emotion regulation by cognitive reappraisal - The role of frontal theta oscillations. NeuroImage. 2013; 81: 412.

[4] Li XB, Luo YJ. The Emotion Effects in the Spatial and Verbal Working Memory: ERP and fMRI Evidence. Advances in Psychological Science. 2011; 19(2): 166.

[5] Suma HN, Murali S. Principal Component Analysis for Analysis and Classification of fMRI activation maps. IJCSNS. 2007; 7(11): 235.

[6] Bai P, Shen HP, Huang XM, Truong Y. A supervised singular value decomposition for independent component analysis of fMRI. Stat Sin. 2008; 18(4): 1233.

[7] Rashid B, Damargju E, Pearlson GD, Callhoun VD. Dynamic connectivity states estimated from resting fMRI Identify differences among Schizophrenia, bipolar disorder, and healthy control subjects. Front Hum Neurosci. 2014; 8: 897.

[8] Windischberger C, Barht M, Lamm C, Schroeder L, Bauer H, Gur RC, et al. Fuzzy cluster analysis of high-field functional MRI data. Artif Intell Med. 2003; 29(3): 203. 
[9] Liu X, Zhu XH, Qiu PH, Chen W. A correlation-matrix-based hierarchical clustering method for functional connectivity analysis. J Neurosci Methods. 2012; 211(1): 94.

[10] Jing YS, Zeng WM, Wang NZ, Ren TL, Shi YC, Yin J, et al. GPU-based parallel group ICA for functional magnetic resonance data. Comput Methods Programs Biomed. 2015; 119(1): 9.

[11] Sui J, Adali T, Pearlson GD, Calhoun VD. An ICA-based method for the identification of optimal FMRI features and components using combined group-discriminative techniques. NeuroImage. 2009; 46(1): 73.

[12] Wang JY, Zhang F, Zhou XZ, Shi YC, Luo W. Segmentation of caption region using wavelet transform and K-mean clustering. Journal of Computer-Aided Design \& Computer Graphics. 2006; 18(10): 1508.

[13] Fu DS, Zhou C. Improved K-means algorithm and its implementation based on density. Journal of Computer Applications. 2011; 31(2): 432.

[14] Yedla M, Pathakota SR, Srinivasa TM. Enhancing K-means Clustering Algorithm with Improved Initial Center. IJCSIT. 2010; 1(2): 121

[15] Salman R, Kecman V, Li Q, Strack R, Test E. Fast K-means algorithm clustering. IJCNC. 2011; 3(4): 17.

[16] He HS, DeZonia BE, Mladenoff DJ. An aggregation index (AI) to quantify spatial patterns of landscapes. Landscape Ecology. 2000; 15(7): 591.

[17] Nelson BD, Fitzgerald DA, Klumpp H, Shankmana SA, Phana KL. Prefrontal engagement by cognitive reappraisal of negative faces Original Research Article. Behav Brain Res. 2015; 279: 218.

[18] Wessing I, Rehbein MA, Postert C, Fürniss T, Junghöfer M. The neural basis of cognitive change: Reappraisal of emotional faces modulates neural source activity in a frontoparietal attention network. NeuroImage. 2013; 81: 15.

[19] Belden AC, Luby JL, Pagliaccio D, Barch DM. Neural activation associated with the cognitive emotion regulation of sadness in healthy children. Dev Cogn Neurosci. 2014; 9: 136. 